\title{
Data Acquisition and Processing of Electronic Compass for Mobile Robot Autonomous Navigation
}

\author{
Jingwei QIN \\ department of computer, Jilin Business \\ and Technology College, \\ Changchun, China \\ E-mail: zml@jlu.edu.cn
}

\author{
Yimu GUO \\ department of electrical engineering, \\ Changchun University of Science and \\ Technology, Changchun, China
}

\author{
Miaolei ZHOU \\ department of control science and \\ engineering, Jilin University, \\ Changchun, China
}

\begin{abstract}
Electronic compass is a kind of absolute sensor that can measure the azimuth by use of the earth's magnetic field. Electronic compass is widely applied in mobile robot autonomous navigation. An electronic compass applied to a certain kind of out-door mobile robot is introduced in this paper. Data format of electronic compass is analyzed. Data acquisition method in Linux operating system is presented in detail. Experimental results show that the proposed method can obtain stable angle data, can meet the navigation demand of outdoor autonomous mobile robot.
\end{abstract}

Keywords-electronic compass; data acquisition; USB communication; robot

\section{Introduction}

In the key technology of outdoor autonomous mobile robot, the autonomous navigation technology is one of the core problems. When outdoor autonomous mobile robot is walking, it must continuously sense the environment information and the state information. A variety of sensors are needed to provide various types of information for autonomous mobile robot [1]-[3]. Thereinto, electronic compass is widely applied in mobile robot autonomous navigation.

Electronic compass, which is also known as digital compass, is a kind of absolute sensor that can measure the azimuth by use of the earth's magnetic field. The angle between the earth's magnetic field vector and sensor will transmit to electronic signal [4], [5]. By processing the electric signal, the direction can be measured by electronic compass. Electronic compass, which plays an important role in the navigation area, provides the direction and status of motorizedtarget. It has already been widely applied in aviation, navigation, vehicle location, geology exploration, and some other fields.

Electronic compass (Model AMS0805WAH, Amosnese production, made in Korea) is selected as the orientation sensor for an outdoor autonomous mobile robot. The sensor configuration of the robot is shown in Figure 1. Robot platform is the Pioneer2-AT robot (American, ActivMedia company). This electronic compass is located at the top of the autonomous mobile robot, and it is used to detect the movement direction of the robot.

\section{Electronic Compass Data Format}

There are 25 bytes involved in electronic compass data including azimuth angle, roll angle, pitch angle and so on. The first three bytes of data are 'A', 'M', 'S', if the first three are accurately received. It indicates that this data is valid. Each two-byte data following the first three indicates one parameter: the first one byte restores high 8 bit data; another byte contains low 8 bit data. Detailed instructions are followed in table 1 .

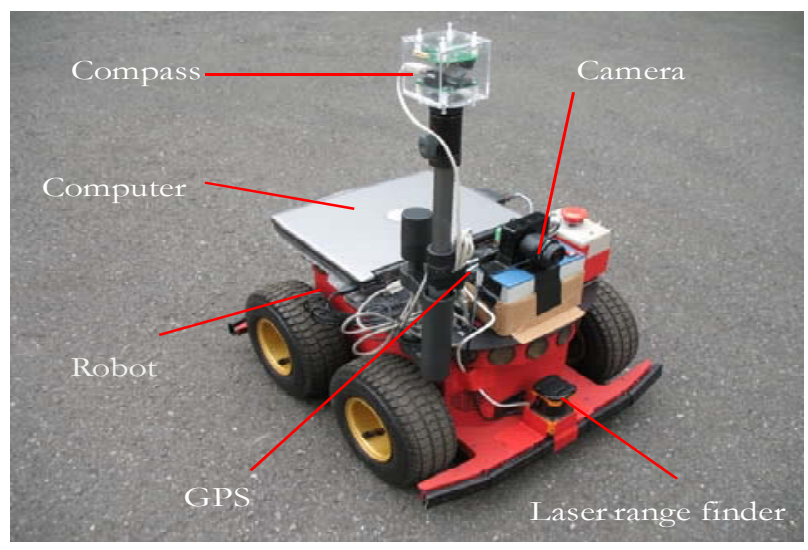

Figure 1. Autonomous mobile of outdoor robot

Table 1. Output data format of electronics compass

\begin{tabular}{ll}
\hline Byte & The data are representative of \\
0 & A \\
1 & M \\
2 & S \\
3 & low byte of azimuth data \\
4 & high byte of azimuth data \\
$\ldots \ldots$ & $\ldots \ldots$ \\
$\ldots \ldots$ & $\ldots \ldots .$. \\
21 & low byte of pitch angle data \\
22 & high byte of pitch angle data \\
23 & low byte of roll angle data \\
24 & high byte of roll angle data \\
\hline
\end{tabular}




\section{Software Implement}

\section{A USB Port Communication}

Controlling computer of robot adopts Linux as operating system. The electronic compass communicates with MCU via USB port. Instructions are followed as below:

1) Opening USB Port

By accessing serial port to USB port converter, we can receive data from electronic compass. The device file can be opened by standard file ‘open’ function.

\section{2) Setting USB Communication Mode}

To adjust communication mode, we mainly set the members in struct termios. The basic settings include baud rate, parity bit, stop bit, etc.

3) Reading from USB Port

By using "read" function in standard file.

\section{4) Closing USB Port}

By using “close” function in standard file.

\section{B Detailed Realization}

First, define a structure variable to describe data information of the electronic compass:

typedef struct_AMS0805WAH

\{

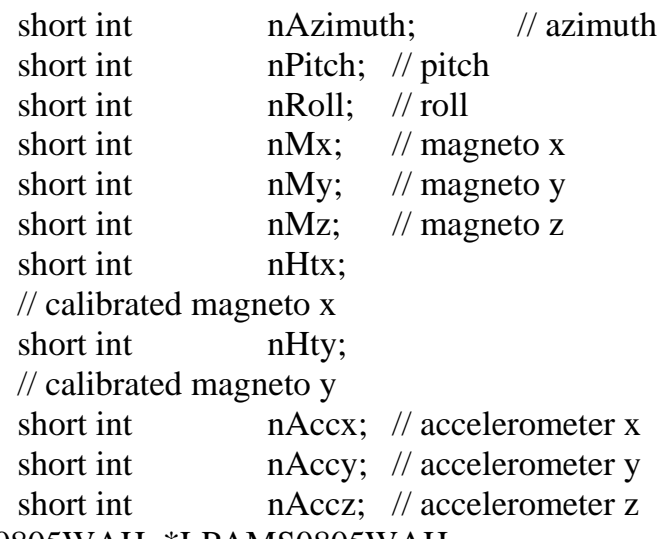

\} AMS0805WAH, *LPAMS0805WAH;

According to the data format of the electronic compass, every parameter in the data stack is constructed by two-byte information except first three bytes. One stores high byte and another one stores low byte. Hence, a function name is 'makeint16' that is defined. It can converts 16-hex received data to 10-hex and then combines them to one digit.

This function is defined as in detail:

\#define makeint16(a, b) ( (int) (((unsigned char) ((intptr_t) (b) \& 0xff)) $\mid$ ((int)((unsigned char) ((intptr_t) (a) \& 0xff)) $<<$ 8)) )

In order to realize data acquisition procedure, we create a set of classes including USB port opening class, USB communication initialization class, electronic compass reading class, etc.
1) USB Opening Class:

It function is realize to open USB port. Name of class is OpenDev.

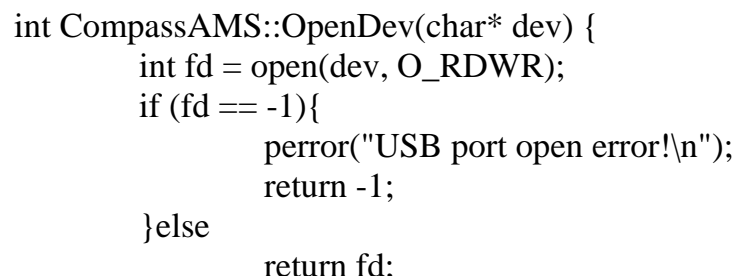

\}

2) USB Communication Initialization Class:

It function is realize to communicate with USB port. Name of class is CompassAMSInit.

void CompassAMS::CompassAMSInit()\{

fd = OpenDev(port);

struct termios opt;

tcgetattr(fd, \&opt);

cfsetispeed(\&opt, B38400);

cfsetospeed(\&opt, B38400);

opt.c_cflag $\mid=($ CLOCAL | CREAD);

opt.c_cflag $\&=\sim \mathrm{CSIZE}$;

opt.c_cflag $\mid=$ CS8; $\quad$ //8 data size

opt.c_cflag \&= CSTOPB; //one

stop bits

opt.c_cflag $\&=\sim$ PARENB; $\quad$ //no

parity

opt.c_lflag $\&=\sim($ ICANON | ECHO | ECHOE | ISIG); opt.c_oflag $\&=\sim$ OPOST;

tcsetattr(fd, TCSANOW, \&opt);

\}

3) Electronic Compass Reading Class

It function is to realize to communicate with USB port. Name of class is ReadCompassData.

int CompassAMS::ReadCompassData (LPAMS0805WAH pAMS) \{

int nread;

unsigned char buff[256];

if $(($ nread $=$ read(fd, buff, 256) $)>0)\{$

buff[nread +1$]=' \backslash 0$ ';

if ( memcmp(buff, "AMS", 3) != 0 )\{ return -1; //Data Reading Failure \}

pAMS->nAzimuth=makeint16(buff[3],buff[4]);

//Azimuth

pAMS->nMx=makeint16(buff[5],buff[6]);

//Magneto $\mathrm{X}$

pAMS->nMy=makeint16(buff[7],buff[8]);

//Magneto Y

pAMS->nMz=makeint16(buff[9],buff[10]);

//Magneto Z

pAMS->nHtx=makeint16(buff[11],buff[12]);

//Calibrated Magneto X

pAMS->nHty=makeint16(buff[13],buff[14]);

//Calibrated Magneto Y

pAMS->nAccx=makeint16(buff[15],buff[16]); 
// Accelerometer X

pAMS->nAccy=makeint16(buff[17],buff[18]);

// Accelerometer Y

pAMS->nAccz=makeint16(buff[19],buff[20]);

// Accelerometer Z

pAMS->nPitch=makeint16(buff[21],buff[22]);

// Pitch

pAMS->nRoll=makeint16(buff[23],buff[24]);

// Roll

return 0;

\}else

return $-1 ;\}$

\section{Experimental Research}

In order to test and verify the electronic compass data acquisition system, and electronic compass data acquisition experiments are researched in this paper.

Obstacle avoidance action happens very frequently when the robot is moving on the road, so the azimuth data from electronic compass is difficult to comply with the direction of the road. In this paper the azimuth data on the straight travel without obstacle avoidance action is chosen as experimental curve of the electronic compass, as the Figure 2 shown. This experiment shows that the acquisition program can collect the azimuth data effectively and stably.

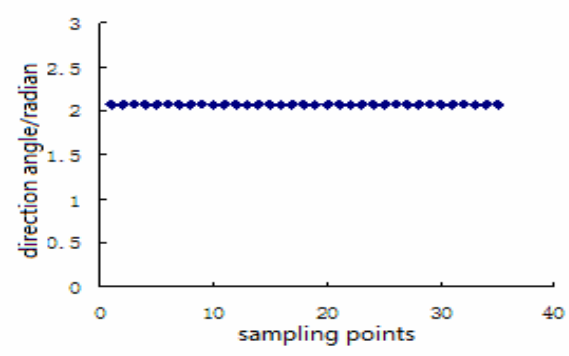

Figure 2. Data acquisition curve of the electronic compass

\section{Conclusion}

For the realization of the robot's autonomous mobile in a complex, dynamic and uncertain environments, a variety of sensors are used to provide all kinds of information the robot needs. Data acquisition method of electronic compass for autonomous mobile outdoor robot is studied in the paper. Data format of electronic compass is analyzed in details. Data acquisition method in Linux operating system is presented. Finally, the experimental result of the robot moving on the road independently is given, and that proved the validity of the data acquisition method.

\section{Acknowledgment}

This research is supported by Program of Science and Technology Development Plan of Jilin province of China (Grant No.: 20090122), Scientific Frontiers and Interdisciplinary Innovation Project of Jilin University of China (Grant No.: 200903309).

\section{References}

[1] M. Yang, H. Wang, K. HE, et al, "Environmental modeling and obstacle avoidance of mobile robots based on laser radar," Journal of Tsinghua University (Sci \&Tech), vol. 40, no. 7, pp. 112-116, 2000.

[2] Ouadah Noureddine, Cadenat Viviane, Lerasle Frédéric, et al, "Multisensor-based control strategy for initiating and maintaining interaction between a robot and a human,” Advanced Robotics, vol. 25, no. 9-10, pp. 1249-1270, 2011.

[3] Lin Chun-Han, King Chung-Ta, "Sensor-deployment strategies for indoor robot navigation," IEEE Transactions on Systems, Man, and Cybernetics Part A:Systems and Humans, vol. 40, no. 2, pp. 388-398, 2010.

[4] Liu Gequn, Liu Weiguo, MA Dongtao, et al, "Application of Electronic Compass Module in Robot Control System,” Chinese Journal of Sensors and Actuators, no. 3, pp. 224-227, 2004.

[5] Hao Zhenhai, Huang Shengguo, "Integrated heading system based on differentialmagnetic compasses," Journal of BeijingUniversity of Aeronautics and Astronautics, vol. 34, no. 4, pp. 377-380, 2008. 